Georgian Mathematical Journal

Volume 14 (2007), Number 4, 687-698

\title{
VECTOR MEASURES ON TOPOLOGICAL SPACES
}

\author{
SURJIT SINGH KHURANA
}

\begin{abstract}
Let $X$ be a completely regular Hausdorff space, $E$ a quasi-complete locally convex space, $C(X)$ (resp. $\left.C_{b}(X)\right)$ the space of all (resp. all, bounded), scalar-valued continuous functions on $X$, and $\mathcal{B}(X)$ and $\mathcal{B}_{0}(X)$ be the classes of Borel and Baire subsets of $X$. We study the spaces $M_{t}(X, E)$, $M_{\tau}(X, E), M_{\sigma}(X, E)$ of tight, $\tau$-smooth, $\sigma$-smooth, $E$-valued Borel and Baire measures on $X$. Using strict topologies, we prove some measure representation theorems of linear operators between $C_{b}(X)$ and $E$ and then prove some convergence theorems about integrable functions. Also, the Alexandrov's theorem is extended to the vector case and a representation theorem about the order-bounded, scalar-valued, linear maps from $C(X)$ is generalized to the vector-valued linear maps.
\end{abstract}

2000 Mathematics Subject Classification: Primary: 46E10, 28C05, 28C15, 46G10; Secondary: 28B05.

Key words and phrases: Strict topologies, vector measures, measure representation of linear operators, Alexandrov's theorem.

\section{Introduction AND Notation}

In this paper $R$ stands for the set of real numbers, $K$ denotes the field of real or complex numbers (we call them scalars) and $X$ a completely regular Hausdorff space and $E$ a quasi-complete locally convex space space over $K$ with topology generated by an increasing family of semi-norms $\|.\|_{p}, p \in P ; E^{\prime}$ denotes the topological dual of $E$. For a $p \in P, V_{p}=\left\{x \in E:\|x\|_{p} \leq 1\right\}$; polars are taken in the duality $\left\langle E, E^{\prime}\right\rangle$. We denote by $C(X)$ the space of all $K$-valued continuous functions on $X$, and by $C_{b}(X)$ the space of all bounded elements of $C(X)$. The zero-sets of $X$ are the elements of $\left\{f^{-1}(0): f \in C_{b}(X)\right\}$; the positive-sets of $X$ are sets of the form $X \backslash Z$ where $Z$ is a zero-set. For locally convex spaces, the notation and results of [9] will be used. For a vector space $F, F^{*}$ will denote its algebraic dual. $N$ will denote the set of natural numbers. For topological measure theory the notation and results of ([10], [11], [5], [12]) will be used. All locally convex spaces are assumed to be Hausdorff and over $K$. The elements of the smallest $\sigma$-algebra, on $X$, relative to which all functions in $C_{b}(X)$ are measurable, are called Baire sets and the elements of the $\sigma$-algebra generated by open sets are called Borel sets. $\mathcal{B}(X)$ and $\mathcal{B}_{0}(X)$ are the classes of Borel and Baire subsets of $X . \tilde{X}$ will denote the Stone-Cech compactification of $X$ and $v X$ the real-compactification. $M_{\sigma}(X), M_{\tau}(X), M_{t}(X)$ denote the spaces of $\sigma$ additive, $\tau$-smooth and tight Baire measures on $X([12],[11])$, respectively. The elements of $M_{\sigma}(X)$ are scalar-valued, countably additive measures on $\mathcal{B}_{0}(X)$. An element $\mu \in M_{\sigma}(X)$ is called $\tau$-smooth if for any decreasing net $\left\{f_{\alpha}\right\} \subset$ 
$C_{b}(X), f_{\alpha} \downarrow 0$, we have $\mu\left(f_{\alpha}\right) \rightarrow 0$. Every $\tau$-smooth measure has a unique extension to a Borel measure which is inner regular by closed subsets and outer regular by open subsets of $X$; an element $\mu \in M_{\sigma}(X)$ is called tight if for any uniformly bounded net $\left\{f_{\alpha}\right\} \subset C_{b}(X), f_{\alpha} \rightarrow 0$, uniformly on the compact subsets of $X$, we have $\mu\left(f_{\alpha}\right) \rightarrow 0$. Every tight measure has a unique extension to a Borel measure which is inner regular by compact subsets and outer regular by open subsets of $X$ ([12], [11]). Also, the so-called strict topologies $\beta_{z}, z=\sigma, \tau, t$ are defined on $C_{b}(X)$, with the result that $\left(C_{b}(X), \beta_{z}\right)^{\prime}=M_{z}(X)$ (see [11]) (notation like $\beta_{1}, \beta, \beta_{0}$ is also used for these topologies in [10]). The topology $\beta_{t}$ is the finest locally convex one on $C_{b}(X)$, agreeing with the topology of uniform convergence on the compact subsets of $X$, on the norm bounded subsets of $C_{b}(X)$. To define the topology $\beta_{\sigma}$, take a zero-set in $\tilde{X}, Z \subset \tilde{X} \backslash X$. The topology $\beta_{t}$ on $C_{b}(\tilde{X} \backslash Z)$ is denoted by $\beta_{Z}$. Evidently, $C_{b}(\tilde{X} \backslash Z)$ can be identified with $C_{b}(X)$ (there is a natural one-to-one, onto, norm-preserving mapping) and so $\beta_{Z}$ can be considered a locally convex topology on $C_{b}(X)$. The topology $\beta_{\sigma}$ is defined as $\bigwedge\left\{\beta_{Z}: Z\right.$ a zero-set in $\left.\tilde{X}, Z \subset \tilde{X} \backslash X\right\}$. Similarly, $\beta_{\tau}$ is defined as $\bigwedge\left\{\beta_{C}: C\right.$ a compact set in $\left.\tilde{X}, C \subset \tilde{X} \backslash X\right\}$.

With norm topology on $C_{b}(X)$, the dual of $C_{b}(X)$ is denoted by $M(X) ; M(X)$ can also be interpreted as the space of bounded finitely additive measures on the algebra generated by zero-sets of $X$, which are inner regular by zero-sets and outer regular by the positive-sets of $X$ (Alexandrov Theorem [12], [11]).

For a function $f \in C_{b}(X), \tilde{f}$ denotes its unique continuous extension to $\tilde{X}$. It can be easily verified that $\mathcal{B}(\tilde{X}) \cap X=\mathcal{B}(X)$ and $\mathcal{B}_{0}(\tilde{X}) \cap X=\mathcal{B}_{0}(X)$.

Now we come to vector-valued measures; the integrability of scalar-valued functions is taken in the sense of ([7]). If $\mathcal{A}$ is a $\sigma$-algebra of subsets of a set $Y, \mu: \mathcal{A} \rightarrow E$ a countably additive vector measure and $p \in P$, we denote the $p$-semi-variation of $\mu$ by $\bar{\mu}_{p}, \bar{\mu}_{p}(A)=\sup \left\{|g \circ \mu|(A): g \in V_{p}^{0}\right\}$ (here $V_{p}^{0}$ is the polar of $V_{p}$ in the duality $\left\langle E, E^{\prime}\right\rangle$ ) [7]; we also consider the submeasure $\dot{\mu}_{p}: \mathcal{A} \rightarrow R^{+}, \dot{\mu}_{p}(A)=\sup \left\{\|\mu(B)\|_{p}: B \in \mathcal{A}, B \subset A\right\}$ ([5], [3]). It is easy to verify that $\dot{\mu}_{p}$ is countably sub-additive [3] and $\dot{\mu}_{p} \leq \bar{\mu}_{p} \leq 4 \dot{\mu}_{p}$. Also, there is a control measure for $\bar{\mu}_{p}$ to be denoted by $\lambda_{p}$; this control measure can be chosen in the closed convex hull of $\left\{|g \circ \mu|: g \in V_{p}^{0}\right\}$, with norm topology on measures ([7], p. 20, the proof of Theorem 1). This control measure also has the following properties: (i) $|f \circ \mu| \ll \lambda_{p}$ for every $f \in E^{\prime}$ with $\|f\|_{p} \leq 1$ (note that $\|f\|_{p}=\sup \left\{|f(x)|: x \in V_{p}\right\}$ ); (ii) if $\lambda_{p}(A)=0$, then $\bar{\mu}_{p}(A)=0$; (iii) $\lim _{\lambda_{p}(A) \rightarrow 0} \bar{\mu}_{p}(A)=0$; (iv) $\lambda_{p} \leq \bar{\mu}_{p}$. We also establish that if $f: Y \rightarrow K$ is a measurable function, $B \in \mathcal{A}$ and $|f| \leq c$ on $B$, then $\left\|\int_{B} f d \mu\right\|_{p} \leq c \bar{\mu}_{p}(B)$.

$L^{1}(\mu)$ denotes the space of $\mu$-integrable functions $([7])$. For any $f \in L^{1}(\mu)$, we take $\bar{\mu}_{p}(f)=\sup \left\{|g \circ \mu|(|f|): g \in V_{p}^{0}\right\}([7]$, Lemma 2, p. 23).

If $\mathcal{F}$ is an algebra of subsets of a set $Y$ and $\mu: \mathcal{F} \rightarrow E$ a finitely additive measure, then $\mu$ is called exhaustive if for any disjoint sequence $\left\{A_{n}\right\} \subset \mathcal{F}$, we have $\mu\left(A_{n}\right) \rightarrow 0$; exhaustive measures are called strongly bounded measures in [2]; for quasi-complete $E$, a finitely additive $\mu$ is exhaustive if and only if $\mu(\mathcal{F})$ 
is relatively weakly compact in $E$ (for Banach spaces, it is proved in [2] and can be easily extended to quasi-complete locally convex spaces).

If $X$ is a compact Hausdorff space then there is a one-to-one correspondence between regular Borel $E$-valued measures $\mu$ and linear weakly compact operators $T: C(X) \rightarrow E$ such that $T(f)=\int f d \mu, \forall f \in C(X)$ ([8], Theorem 3.1, p. 163); regularity means that for any Borel $B \subset X, p \in P$, and $c>0$, there exist a compact $C$ and an open $\mathrm{V}, C \subset B \subset V$ such that $\bar{\mu}_{p}(V \backslash C)<c$. In that case, for $p \in P$, the control measure $\lambda_{p}$ is a positive regular Borel measure in $X$.

In this paper, by taking the strict topologies on $C_{b}(X)$ we get similar representation theorems for weakly compact and continuous linear maps from $C_{b}(X)$ into $E$. Some convergence type theorems having relevance to topology are also proved. With a norm topology on $C_{b}(X)$, the celebrated Alexandrov's theorem says that the dual of $C_{b}(X)$ is $M(X)$; we extend this result to weakly compact and continuous linear $\mu: C_{b}(X) \rightarrow E$. Another very well-known result in the scalar case is that a linear $\mu: C(X) \rightarrow R$, which maps order bounded subsets into bounded sets, comes from a countably additive $\mu \in M_{\sigma}(X)$, whose support is in $v X$; this result is also extended to linear maps $\mu: C(X) \rightarrow E$.

First we consider $X$ to be a compact Hausdorff space and prove some properties of $E$-valued regular Borel measures on it; then we extend these properties to completely regular Hausdorff spaces.

\section{Representation Theorems}

Theorem 1. Let $X$ be a compact Hausdorff space and $\mu$ an E-valued regular Borel measure on $X$.

(i) Suppose $\left\{f_{\alpha}\right\}$ is an increasing net of non-negative, lower semi-continuous functions in $L^{1}(\mu)$, converging to $f \in L^{1}(\mu)$, pointwise on $X$. Then $\lim \bar{\mu}_{p}(f-$ $\left.f_{\alpha}\right)=0$; in particular $\lim \int f_{\alpha} d \mu=\int f d \mu$.

(ii) Given a $p \in P$, there exists the largest open set $U_{p} \subset X$ such that $\bar{\mu}_{p}\left(U_{p}\right)=$ 0 ; this $X \backslash U_{p}$ is called the support of $\bar{\mu}_{p}$ and has the property that for any $f \in C_{b}(X), f \geq 0$, and $f$ not identically 0 on $X \backslash U_{p}$, one has $\bar{\mu}_{p}(f)>0$.

Proof. (i) Fix a $p \in P$ and let $\lambda_{p}$ be the corresponding control measure. Since $\lambda_{p}$ is in the norm-closed, absolutely convex hull of $\left\{|g \circ \mu|: g \in E^{\prime},\|g\|_{p} \leq 1\right\}$, it follows that $f$ is $\lambda_{p}$-integrable. As $\lambda_{p}$ is a regular Borel measure, $\lim \int f_{\alpha} d \lambda_{p}=$ $\int f d \lambda_{p}$. This means there are an increasing sequence $\left\{f_{\alpha(n)}\right\}$ and a Borel $B \subset X$ such that $\lambda_{p}(X \backslash B)=0$ and $f_{\alpha(n)} \rightarrow f$ pointwise on $B$. Using the fact $f_{\alpha(n)} \leq f, \forall n$, by $\left([7]\right.$, Theorem 1, p. 20), $\bar{\mu}_{p}\left(f-f_{\alpha(n)}\right) \rightarrow 0$. This proves this result.

(ii) Fix a $p \in P$ and let $\mathcal{V}=\left\{U \subset X: U\right.$ open and $\left.\bar{\mu}_{p}(U)=0\right\}$. By the subadditivity of $\bar{\mu}_{p}$, for any finite collection $\left\{U_{i}(1 \leq i \leq n)\right\} \subset \mathcal{V}, \bar{\mu}_{p}\left(\cup U_{i}\right)=0$. From (i) $\bar{\mu}_{p}(\cup\{U: U \in \mathcal{V}\})=0$. The other statement is easy to prove.

Now assume that $X$ is a completely regular Hausdorff space and $\mathcal{B}(X)$ and $\mathcal{B}_{0}(X)$ be the classes of Borel and Baire subsets of $X([11])$. If it is not necessary to specify the space $X$, we will also denote them by $\mathcal{B}$ and $\mathcal{B}_{0}$. Let $M_{\sigma}(X, E)=$ 
$\left\{\left(\mu: \mathcal{B}_{0} \rightarrow E\right): g \circ \mu \in M_{\sigma}(X), \forall g \in E^{\prime}\right\}$. This implies that every $\mu \in$ $M_{\sigma}(X, E)$ is countably additive in the original topology of $E$.

Theorem 2. Suppose $X$ is a completely regular Hausdorff space and $\mu \in$ $M_{\sigma}(X, E)$ is a countably additive Baire measure. Then

(i) $\mu$ is inner regular by zero-sets and outer regular by positive sets;

(ii) the linear mapping $\mu:\left(C_{b}(X), \beta_{\sigma}\right) \rightarrow E$ is continuous and bounded sets are mapped into relatively weakly compact sets.

Conversely, if a linear mapping $\mu:\left(C_{b}(X), \beta_{\sigma}\right) \rightarrow E$ is continuous and maps bounded sets into relatively weakly compact sets, then there exists a unique countably additive Baire measure $\nu: \mathcal{B}_{0} \rightarrow E$ such that $\int f d \nu=\mu(f), \forall f \in$ $C_{b}(X)$.

Proof. (i) Note $\mathcal{B}_{0}(\tilde{X}) \cap X=\mathcal{B}_{0}(X)$. Define $\tilde{\mu}: \mathcal{B}_{0}(\tilde{X}) \rightarrow E, \tilde{\mu}(B)=\mu(B \cap X)$. This means $\tilde{\mu}(B)=0$ when $B \cap X=\varnothing$. Take a $p \in P, c>0$ and a Baire set $B \subset X$. Select a Baire $\tilde{B} \subset \tilde{X}$ such that $\tilde{B} \cap X=B$. Since a Baire measure on a compact Hausdorff space is regular ([5]), there exists a zero-set $Z$ and a positive set $V$ in $\tilde{X}$ such that $Z \subset \tilde{B} \subset V$ and $\overline{\tilde{\mu}}_{p}(V \backslash Z) \leq c$. From this it follows that $\bar{\mu}_{p}(V \cap X \backslash Z \cap X) \leq c$. This proves the regularity of $\mu$.

(ii) Since the range of a countably additive $E$-valued measure is a relatively weakly compact subset of $E$, the unit ball of $C_{b}(X)$ is mapped into a relatively weakly compact subset of $E$ under the mapping $\mu:\left(C_{b}(X), \beta_{\sigma}\right) \rightarrow E$. Also $\beta_{\sigma}$-bounded sets are norm-bounded $([11])$ and so the bounded sets are mapped into a relatively weakly compact subset of $E$.

Now for every $g \in E^{\prime}, g \circ \mu \in M_{\sigma}(X)$ and so, with weak topology on $E$, the mapping $\mu:\left(C_{b}(X), \beta_{\sigma}\right) \rightarrow E$ is continuous. Since $\beta_{\sigma}$ is Mackey ([11]), the mapping is also continuous with the original topology on $E([9], 7.4$, p. 149).

Conversely, suppose that $\mu:\left(C_{b}(X), \beta_{\sigma}\right) \rightarrow E$ is a linear and continuous mapping and the bounded sets are mapped into a relatively weakly compact subset of $E$. With sup-norm topology on $C(\tilde{X})$, the mapping $\tilde{\mu}: C(\tilde{X}) \rightarrow E$, $\tilde{\mu}(f)=\mu\left(f_{\mid X}\right), \forall f \in C(\tilde{X})$, is linear and weakly compact and so $\tilde{\mu}$ can be considered a regular Baire measure on $\tilde{X}$. If $Z \subset \tilde{X} \backslash X$ is a zero-set, there exists a sequence $\left\{f_{n}\right\} \subset C(\tilde{X})$ such that $f_{n} \downarrow \chi_{Z}$. This means, in $\left(C_{b}(X), \beta_{\sigma}\right)$, $f_{n \mid X} \rightarrow 0$. Thus for every zero-set $Z \subset \tilde{X} \backslash X, \tilde{\mu}(Z)=0$, and so, for every $p \in P, \overline{\tilde{\mu}}_{p}(B)=0$, for all Baire sets $B \subset \tilde{X} \backslash X$. For any Baire set $A \subset X$, define $\nu(A)=\tilde{\mu}(B), B$ being any Baire subset of $\tilde{X}$, with $B \cap X=A$. It is a routine verification that $\nu$ is well-defined, is countably additive and for the integration of any $f \in C_{b}(X), \int f d \nu=\int f d \mu$. Also if there is another Baire measure $\nu_{1}$, on $X$, such that $\int f d \nu=\int f d \nu_{1}$ for every $f \in C_{b}(X)$, then we have $\nu(Z)=\nu_{1}(Z)$ for every zero-set $Z \subset X$; by regularity, this will imply $\nu=\nu_{1}$. So the uniqueness is established.

A Baire measure $\mu: \mathcal{B}_{0} \rightarrow E$ is called $\tau$-smooth if for every $g \in E^{\prime}, g \circ \mu \in$ $M_{\tau}(X)$. The set of all $E$-valued $\tau$-smooth measures is denoted by $M_{\tau}(X, E)$.

Theorem 3. Suppose $X$ is a completely regular Hausdorff space and $\mu$ : $\mathcal{B}_{0} \rightarrow E$ is a $\tau$-smooth measure. Then 
(i) $\mu$ can be extended to a Borel measure which is inner regular by closed sets and outer regular by open sets (we call this extension a regular Borel Measure);

(ii) the linear mapping $\mu:\left(C_{b}(X), \beta_{\tau}\right) \rightarrow E$ is continuous and bounded sets are mapped into relatively weakly compact sets;

(iii) considering $\mu$ a Borel measure, suppose $\left\{f_{\alpha}\right\}$ is an increasing net of nonnegative, lower semi-continuous functions in $L^{1}(\mu)$, converging pointwise to an $f \in L^{1}(\mu)$. Then $\lim \bar{\mu}_{p}\left(f-f_{\alpha}\right)=0$; in particular $\lim \int f_{\alpha} d \mu=\int f d \mu$.

(iv) Given a $p \in P$, there exists the largest open set $U_{p} \subset X$ such that $\bar{\mu}_{p}\left(U_{p}\right)=0$; this $X \backslash U_{p}$ is called the support of $\bar{\mu}_{p}$ and has the property that for any $f \in C_{b}(X), f \geq 0$, and $f$ not identically 0 on $X \backslash U_{p}$, one has $\bar{\mu}_{p}(f)>0$.

(v) The Borel regular extension of $\mu$, satisfying the condition that, for an increasing net $\left\{V_{\alpha}\right\}$ of open subsets of $X$ with $\cup V_{\alpha}=V$, we have $\lim \mu\left(V_{\alpha}\right)=$ $\mu(V)$, is unique.

Conversely, if a linear mapping $\mu:\left(C_{b}(X), \beta_{\tau}\right) \rightarrow E$ is continuous and maps bounded sets into relatively weakly compact sets, then there exists a unique $\tau$ smooth measure $\nu: \mathcal{B}_{0} \rightarrow E$ such that $\int f d \nu=\mu(f), \forall f \in C_{b}(X)$.

Proof. (i). We have $\mathcal{B}(\tilde{X}) \cap X=\mathcal{B}(X)$. As $C_{b}(X) \subset L^{1}(\mu)$, we get a linear continuous $\tilde{\mu}: C(\tilde{X}) \rightarrow E, \tilde{\mu}(f)=\mu\left(f_{\mid X}\right), \forall f \in C(\tilde{X})$. Thus $\tilde{\mu}$ can be considered as a regular Borel measure on $\tilde{X}$. Take a closed set $C \subset \tilde{X} \backslash X$; there exists a net $\left\{f_{\alpha}\right\} \subset C(\tilde{X})$ such that $f_{\alpha} \downarrow \chi_{C}$. This means, in $\left(C_{b}(X), \beta_{\tau}\right)$, $f_{\alpha \mid X} \rightarrow 0$. Thus for every closed set $C \subset \tilde{X} \backslash X, \tilde{\mu}(C)=0$, and so, by regularity, for every $p \in P, \overline{\tilde{\mu}}_{p}(B)=0$, for all Borel sets $B \subset \tilde{X} \backslash X$. For any Borel set $A \subset X$, define $\nu(A)=\tilde{\mu}(B)$, B being any Borel subset of $\tilde{X}$, with $B \cap X=A$. It is a routine verification that $\nu$ is well-defined, is countably additive and for the integration of any $f \in C_{b}(X)$ we have $\int f d \nu=\int f d \mu$. Also by the regularity of $\tilde{\mu}$ it can be easily verified that $\mu$ is inner regular by closed sets and outer regular by open sets.

(ii) To prove the continuity of $\mu:\left(C_{b}(X), \beta_{\tau}\right) \rightarrow E$, we get $\tilde{\mu}: C(\tilde{X}) \rightarrow E$ as done above. Fix a $p \in P$, put $\mathrm{M}=\overline{\tilde{\mu}}_{p}(\tilde{X})$, and fix an $n \in N$. Take a compact $C \subset \tilde{X} \backslash X$. Now the topology $\beta_{C}$ is identical with the topology $\beta_{t}$ on $C_{b}(\tilde{X} \backslash C)$, if we identify $C_{b}(X)$ with $C_{b}(\tilde{X} \backslash C)([11])$. Thus it is enough to prove that $\tilde{\mu}:\left(C_{b}(\tilde{X} \backslash C), \beta_{t}\right) \rightarrow E$ is continuous. We will use the fact that $\beta_{t}$ is the finest locally convex topology agreeing with the compact-open topology on normbounded sets. Take a compact $K \subset \tilde{X} \backslash C$ such that $\overline{\tilde{\mu}}_{p}((\tilde{X} \backslash C) \backslash K) \leq \frac{1}{3 n}$. Since $\tilde{\mu}_{p}(C)=0$, we have $\overline{\tilde{\mu}}_{p}(\tilde{X} \backslash K) \leq \frac{1}{3 n}$. Take an $f \in C_{b}(X),|f| \leq n,|\tilde{f}| \leq \frac{1}{2 n M}$ on K. Now $\int \tilde{f} d \tilde{\mu}=\int_{K} \tilde{f} d \tilde{\mu}+\int_{\tilde{X} \backslash K} \tilde{f} d \tilde{\mu}$. Taking the $\|\cdot\|_{p}$-norm on both sides, we get $\|\mu(f)\|_{p} \leq \frac{1}{2 n M} M+\frac{1}{3 n} n \leq 1$. This proves the continuity of $\mu$.

(iii) Since $g \circ \mu \in M_{\tau}(X), \forall g \in E^{\prime}$, we get that the control measure $\lambda_{p} \in$ $M_{\tau}(X)$. As in Theorem 1, this means $\lim \int f_{\alpha} d \lambda_{p}=\int f d \lambda_{p}$. So we get an increasing sequence $f_{\alpha(n)}$ and a Borel $B \subset X$ such that $\lambda_{p}(X \backslash B)=0$ and $f_{\alpha(n)} \rightarrow f$ pointwise on $B$. Using the fact $f_{\alpha(n)} \leq f, \forall n$, by ([7], Theorem $1, \mathrm{p}$. 20), $\bar{\mu}_{p}\left(f-f_{\alpha(n)}\right) \rightarrow 0$. This proves the result.

(iv) The proof is identical to the one given in Theorem 1 (ii). 
(v) Suppose $\nu_{1}$ and $\nu_{2}$ are two regular Borel extensions of $\nu$, satisfying the given condition. Fix an open set $V \subset X$ and take an increasing net $\left\{U_{\alpha}\right\}$ of positive-sets in $X$ such that $U_{\alpha} \uparrow V$. By (iii) $\nu_{1}(V)=\nu_{2}(V)$ and so, by regularity, $\nu_{1}=\nu_{2}$.

Conversely, suppose that $\mu:\left(C_{b}(X), \beta_{\tau}\right) \rightarrow E$ is a linear and continuous mapping and the bounded sets are mapped into relatively weakly compact subset of $E$. Proceeding as in Theorem 2, we get a unique countably additive Baire measure $\nu$ on $X$ such that $\int f d \nu=\mu(f)$, for every $f \in C_{b}(X)$. Now for every $g \in E^{\prime}, g \circ \mu:\left(C_{b}(X), \beta_{\tau}\right) \rightarrow K$ is a linear and continuous and $g \circ \mu \in M_{\tau}(X)$. This means $\nu$ is $\tau$-smooth.

A countably additive Baire measure $\mu: \mathcal{B}_{0} \rightarrow E$ is called tight if for every $g \in E^{\prime}, g \circ \mu \in M_{t}(X)$. The set of all $E$-valued tight measures will be denoted by $M_{t}(X, E)$. It is a trivial verification that a tight measure $\mu: \mathcal{B}_{0} \rightarrow E$ is also $\tau$-smooth.

Theorem 4. Suppose $X$ is a completely regular Hausdorff space and $\mu$ : $\mathcal{B}_{0} \rightarrow E$ is a tight measure. Then

(i) $\mu$ can be extended to a Borel measure which is inner regular by compact sets and outer regular by open sets;

(ii) the linear mapping $\mu:\left(C_{b}(X), \beta_{t}\right) \rightarrow E$ is continuous and bounded sets are mapped into relatively weakly compact sets;

(iii) considering $\mu$ a Borel measure, suppose $\left\{f_{\alpha}\right\}$ is an increasing net of nonnegative, lower semi-continuous functions in $L^{1}(\mu)$, converging pointwise to an $f \in L^{1}(\mu)$, pointwise on $X$. Then $\lim \bar{\mu}_{p}\left(f-f_{\alpha}\right)=0, \forall p \in P$; in particular $\lim \int f_{\alpha} d \mu=\int f d \mu$;

(iv) the regular Borel extension of $\mu$, satisfying condition (i), is unique.

Conversely, if a linear mapping $\mu:\left(C_{b}(X), \beta_{t}\right) \rightarrow E$ is continuous and maps bounded sets into relatively weakly compact sets, then there exists a unique tight measure $\nu: \mathcal{B}_{0} \rightarrow E$ such that $\int f d \nu=\mu(f), \forall f \in C_{b}(X)$.

Proof. (i). Since the measure is $\tau$-smooth, using Theorem 3, it can be uniquely extended to a Borel measure, satisfying condition (iii) of Theorem 3. Now considering this a Borel measure and using the fact for every $g \in E^{\prime}, g \circ \mu \in$ $M_{t}(X)$, we get that $\mu$ is regular in the weak topology on $E$. By ([8], Theorem 1.6, p. 159), $\mu$ is inner regular by compact subsets of $X$; it is a simple verification that this implies that $\mu$ is outer regular by open subsets of $X$.

(ii) To prove the continuity of $\mu:\left(C_{b}(X), \beta_{t}\right) \rightarrow E$, we will use the fact that $\beta_{t}$ is the finest locally convex topology agreeing with the compact-open topology on norm-bounded sets. Fix a $p \in P$, an $n \in N$ and a $c>0$. Take an $M>0$ such that $\bar{\mu}_{p}(X) \leq M$. Take a compact $C \subset X$ such that $\bar{\mu}_{p}(X \backslash C) \leq \frac{1}{2 n}$. Now for any $f \in C_{b}(X),\|f\| \leq n$ and $|f| \leq \frac{1}{2 M}$ on $C$ we have $\mu(f)=\int_{C} f d \mu+\int_{X \backslash C} f d \mu$. Taking the $\|\cdot\|_{p}$-norm on both sides, we get $\|\mu(f)\|_{p} \leq\left\|\int_{C} f d \mu\right\|_{p}+\left\|\int_{X \backslash C}\right\|_{p} \leq$ $\frac{1}{2 M} M+n \frac{1}{2 n}<1$.

Since $\beta_{t}$ is the finest locally convex topology, agreeing with the compactopen topology on bounded sets, we prove that $\mu$ is continuous. Also, since 
$\mu$ is countably additive, the bounded sets are mapped into relatively weakly compact subsets of $E$.

(iii) Since the measure $\mu$ is $\tau$-smooth, this follows from (iii) of Theorem 3 .

(iv) Let $\mu_{i}(\mathrm{i}=1,2)$ be two Borel extensions of $\mu$, satisfying (i). Take an open $V$ and a compact $C$ in $X$. There is a zero-set $Z$ in $X, C \subset Z \subset V$. Since $\mu_{1}=\mu_{2}$ on zero-sets, we get $\mu_{1}(V)=\mu_{2}(V)$. By regularity, $\mu_{1}=\mu_{2}$.

Conversely, suppose that $\mu:\left(C_{b}(X), \beta_{t}\right) \rightarrow E$ is a linear and continuous mapping and the bounded sets are mapped into relatively weakly compact subsets of $E$. Proceeding as in Theorem 2, we get a unique countably additive Baire measure $\nu$ on $X$ such that $\int f d \nu=\mu(f)$ for every $f \in C_{b}(X)$. Now for every $g \in E^{\prime}, g \circ \mu:\left(C_{b}(X), \beta_{t}\right) \rightarrow K$ is a linear and continuous and so $g \circ \mu \in M_{t}(X)$. This means $\nu$ is tight.

\section{Alexandrov's Theorem}

In this section, we extend the celebrated Alexandrov representation theorem to the vector-valued measures. In the scalar case, in a simple form, this theorem says:

Suppose $X$ is a completely regular Hausdorff space, $\mathcal{F}$ the algebra generated by zero-sets and $\mu: C_{b}(X) \rightarrow K$ a continuous linear mapping. Then there exists a unique, finitely additive measure $\nu: \mathcal{F} \rightarrow R$ such that

(i) $\nu$ is inner regular by zero-sets and outer regular by positive-sets;

(ii) $\int f d \nu=\mu(f), \forall f \in C_{b}(X)$. ([12], Theorem 6, p. 163; [11], ). Note $C_{b}(X)$ is contained in the uniform closure of $\mathcal{F}$-simple functions on $X$ in the space of all bounded functions on $X$ and so each $f \in C_{b}(X)$ is $\nu$-integrable.

We state and prove the following extension. Our proof is obtained by the regularity properties of the corresponding regular Borel measure on $\tilde{X}$ and is very different from that given in [11]. We start with a lemma.

Lemma 5. If $Z_{1}$ and $Z_{2}$ are zero-sets in $X$, then $\overline{Z_{1} \cap Z_{2}}=\overline{Z_{1}} \cap \overline{Z_{2}}$ (for a subset $A \subset X, \bar{A}$ denotes the closure of $A$ in $\tilde{X})$. Hence if $Z_{1} \cap Z_{2}=\varnothing$, then $\overline{Z_{1}} \cap \overline{Z_{2}}=\varnothing$.

Proof. Suppose this is not true. Take a point $a \in \overline{Z_{1}} \cap \overline{Z_{2}} \backslash \overline{Z_{1} \cap Z_{2}}$ (note $Z_{1} \cap Z_{2}$ can be empty). Take an $f \in C_{b}(X), 0 \leq f \leq 1$, such that $\tilde{f}(a)=1$ and $f=0$ on $Z_{1} \cap Z_{2}$. For $i=1,2$, take $h_{i} \in C_{b}(X)$ such that $0 \leq h_{i} \leq 1$ and $Z_{i}=h_{i}^{-1}(0)$. Define $f_{i}(x)=f(x) \frac{h_{i}(x)}{h_{1}(x)+h_{2}(x)}$, for $x \notin Z_{1} \cap Z_{2}$, and 0 otherwise. These functions are continuous and $f=f_{1}+f_{2}$. Thus $\tilde{f}=\tilde{f}_{1}+\tilde{f}_{2}$. Since $f_{i}=0$ on $Z_{i}, \tilde{f}_{i}=0$ on $\overline{Z_{i}}$ and so $\tilde{f}_{1}+\tilde{f}_{2}=0$ on $\overline{Z_{1}} \cap \overline{Z_{2}}$. This means $\tilde{f}(a)=0$, a contradiction.

Now we come to the main theorem.

Theorem 6. Suppose $X$ is a completely regular Hausdorff space and $\mu$ : $C_{b}(X) \rightarrow E$ a weakly compact linear mapping. Then there exists a unique finitely additive, exhaustive measure $\nu: \mathcal{F} \rightarrow E$ such that

(i) $\nu$ is inner regular by zero-sets and outer regular by positive-sets;

(ii) $\int f d \nu=\mu(f), \forall f \in C_{b}(X)$. 
Proof. Considering $\tilde{\mu}: C(\tilde{X}) \rightarrow E$, we get an $E$-valued regular Borel measure $\tilde{\mu}: \mathcal{B}(\tilde{X}) \rightarrow E$. If $A$ is a subset of $X$ or $\tilde{X}, \bar{A}$ will denote the closure of $A$ in $\tilde{X}$. We prove this theorem in several steps.

I. Let $\overline{\mathcal{Z}}=\{\bar{A}: A$ a zero-set in $X\}$. Then for every $Q \in \overline{\mathcal{Z}}$ and $c>0$, there exists $W \in \overline{\mathcal{Z}}$ such that $W \subset \tilde{X} \backslash Q$ and $\overline{\tilde{\mu}}_{p}((\tilde{X} \backslash Q) \backslash W)<c$.

Proof. Using the inner regularity of $\overline{\tilde{\mu}}_{p}$ and Urysohn's lemma, we can take a positive set $V \subset \tilde{X} \backslash Q$ having the property that $\overline{\tilde{\mu}}_{p}((\tilde{X} \backslash Q) \backslash V)<\frac{c}{2}$. Take a $g \in C(\tilde{X}), 0 \leq g \leq 1$, such that $V=g^{-1}(0,1]$. Put $V_{n}=\left\{x \in \tilde{X}: g(x)>\frac{1}{n}\right\}$ and $Z_{n}=\left\{x \in \tilde{X}: g(x) \geq \frac{1}{n}\right\}$. Now, using the fact that $X$ is dense in $\tilde{X}$, we have $V_{n} \subset \overline{\left(V_{n} \cap X\right)} \subset \overline{\left(Z_{n} \cap X\right)} \subset Z_{n} \subset V_{n+1}$ By choosing $n$ sufficiently large we can assume $\overline{\tilde{\mu}}_{p}\left(V \backslash V_{n}\right)<\frac{c}{2}$. Taking $W=\overline{\left(Z_{n+1} \cap X\right)}$, we get the result.

II. Let $\mathcal{A}$ be the algebra, in $\tilde{X}$, generated by $\overline{\mathcal{Z}}$ and denote by $\mathcal{A}_{0}$ the elements of $\mathcal{A}$ which have the property that these elements and their complements are inner regular by the elements of $\overline{\mathcal{Z}}$. Then $\mathcal{A}_{0}=\mathcal{A}$.

Proof. We use I to prove. By I, $\mathcal{A}_{0} \supset \overline{\mathcal{Z}}$. By definition, $\mathcal{A}_{0}$ is closed under complements. Also, using Lemma 5 , it is a routine verification that if $A$ and $B$ are in $\mathcal{A}_{0}$, then $A \cup B$ and $A \cap B$ are also in $\mathcal{A}_{0}$. This proves the result.

III. Let $\mathcal{F}$ be the algebra, in $X$, generated by zero-sets in $X$. Then it is a simple verification that $\mathcal{A} \cap X \supset \mathcal{F}$. Also if $A \in \mathcal{A}$ and $A \cap X=\varnothing$, then $\overline{\tilde{\mu}}_{p}(A)=0$. To prove this, take any $\bar{Z} \in \overline{\mathcal{Z}}, Z$ being a zero-set in $X$, such that $\bar{Z} \subset A$. This means $Z$ is empty and so $\overline{\tilde{\mu}}_{p}(A)=0$. Now we can define a $\nu: \mathcal{F} \rightarrow E, \nu(B)=\tilde{\mu}(A), A$ being any element in $\mathcal{A}$ with $B=A \cap X$; it is a trivial verification that $\nu$ is well-defined, is finitely additive and it is inner regular by zero-sets in $X$ and outer regular by positive-sets in $X$. We also have $\nu(Z)=\tilde{\mu}(\bar{Z})$ for any zero-set $Z \subset X$. Since $\nu(\mathcal{F})$ is relatively weakly compact in $E, \nu$ is exhaustive (三strongly additive) ([2], Corollary 3, p. 28; this is proved for Banach space $E$, but easily extends to the quasi-complete locally convex space $E)$. Also, for any $B \in \mathcal{F}, \bar{\nu}_{p}(B) \leq \overline{\tilde{\mu}}_{p}(A)$, where $A$ is any element in $\mathcal{A}$ such that $B=A \cap X$.

IV. For any $f \in C_{b}(X), \mu(f)=\int f d \nu$.

Proof. Assume $\overline{\tilde{\mu}}_{p}(X) \leq 1$. Fix a $c>0$ and take an $f \in C_{b}(X), 0 \leq f \leq 1$. Then there is a non-negative, $\mathcal{F}$-simple function $\sum_{i=1}^{n} a_{i} \chi_{B_{i}}$ such that $B_{i}$ 's are mutually disjoint, their union is $X$ and $\left|f-\sum_{i=1}^{n} a_{i} \chi_{B_{i}}\right|<c$ on $X$. Take mutually disjoint $\left\{A_{i}\right\} \subset \mathcal{A}$ such that $B_{i}=A_{i} \cap X$ for every $i$. Also take mutually disjoint zero-sets $\left\{Z_{i}\right\} \subset X$ such that $\overline{\tilde{\mu}}_{p}\left(A_{i} \backslash \overline{Z_{i}}\right)<\frac{c}{n}$, for each $i$. Now

$$
\begin{gathered}
\left\|\int f d \nu-\sum a_{i} \nu\left(Z_{i}\right)\right\|_{p} \leq\left\|\int f d \nu-\sum a_{i} \nu\left(B_{i}\right)\right\|_{p}+\left\|\sum a_{i} \nu\left(B_{i} \backslash Z_{i}\right)\right\|_{p} \\
\leq c+\left\|\sum a_{i} \tilde{\mu}\left(A_{i} \backslash \overline{Z_{i}}\right)\right\|_{p} \leq c+n \frac{c}{n}=2 c .
\end{gathered}
$$

Also, $\left|f-\sum_{i=1}^{n} a_{i} \chi_{B_{i}}\right| \leq c$ implies that $\left|\tilde{f}-\sum_{i=1}^{n} a_{i} \chi_{\overline{\left(Z_{i}\right)}}\right| \leq c$ on $\cup\left(\overline{Z_{i}}\right)$ (note $\overline{Z_{i}}$ are also mutually disjoint by Lemma 5). So $\left\|\int \tilde{f} d \tilde{\mu}-\sum a_{i} \nu\left(Z_{i}\right)\right\|_{p}=\| \int \tilde{f} d \tilde{\mu}-$ 
$\sum a_{i} \tilde{\mu}\left(\overline{Z_{i}}\right)\left\|_{p} \leq c+\right\| \sum a_{i} \tilde{\mu}\left(A_{i} \backslash \overline{Z_{i}}\right) \|_{p} \leq c+n \cdot \frac{c}{n}=2 c$. This prove that $\mu(f)=$ $\nu(f)$.

V. Uniqueness.

Proof. Let $\nu: \mathcal{F} \rightarrow E$ be a finitely additive regular (inner regular by zerosets in $X$ and outer regular by positive-sets in $X$ ) measure, having a relatively weakly compact range, such that $\int f d \nu=0, \forall f \in C_{b}(X)$. This means $\nu$ is exhaustive and so $\bar{\nu}_{p}(X)<\infty, \forall p \in P$. If $\nu \neq 0$, then there is $p \in P$, a zero-set $Z \subset X$, and a $c>0$ such that $\|\nu(Z)\|_{p}=2 c$. Take a a positive-set $U \supset Z$ such that $\bar{\nu}_{p}(U \backslash Z)<c$. Then take an $f \in C_{b}(X), 0 \leq f \leq 1$, $f(Z)=\{1\}, f(X \backslash U)=\{0\}$. We get $0=\int f d \nu=\int_{Z} f d \nu+\int_{U \backslash Z} f d \nu$. This means $\nu(Z)=-\int_{U \backslash Z} f d \nu$ and so $2 c \leq 1 . \bar{\nu}_{p}(U \backslash Z)<c$. This contradiction proves the uniqueness.

We denote by $M(X, E)$ the set of all exhaustive, finitely additive $\nu: \mathcal{F} \rightarrow E$ which are inner regular by zero-sets in $X$ and outer regular by positive-sets in $X$; they are the collection of all weakly compact, continuous linear maps $\nu: C_{b}(X) \rightarrow E$.

\section{Representation Theorem for $C(X)$ with a Completely REGULAR $X$}

In this section we assume that $K=R$. A subset $B \subset C(X)$ will be called order-bounded if there are elements $f$ and $g$ in $C(X)$ such that $f \leq b \leq g, \forall b \in$ $B$. It is well-known that a linear map $\mu: C(X) \rightarrow R$, which maps orderbounded sets into bounded sets, gives a unique $\nu \in M_{\sigma}(X)$ such that $C(X) \subset$ $L^{1}(\nu)$ and $\mu(f)=\int f d \nu([12]$, Theorem $23 ;[4])$.

We will extend this fact to the vector case.

Theorem 7. Let $\mu: C(X) \rightarrow E$ be a linear map such that order-bounded subsets are mapped into relatively weakly compact subsets of $E$. Then

(i) There is a unique $\nu \in M_{\sigma}(X, E)$ such that $C(X) \subset L^{1}(\nu)$ and $\mu(f)=$ $\int f d \nu$;

(ii) for every $p \in P$ there is compact $C \subset v X$ (the real-compactification of $X)$, depending on $p$, such that $\overline{\tilde{\nu}}_{p}(\tilde{X} \backslash C)=0([4])$.

Proof. (i) We will use the fact that, when $E=R$, the result is known. First restrict $\mu$ to $C_{b}(X)$; this means $\mu$ is a weakly compact linear operator and $\forall h \in E^{\prime}, h \circ \mu \in M_{\sigma}(X)$ (here we are using the fact that, for $E=R$, the result is known). So there exists a $\nu \in M_{\sigma}(X, E)$ such that $\mu(f)=\int f d \nu, \forall f \in C_{b}(X)$ and $C(X) \subset L^{1}(|h \circ \nu|)$ for every $h \in E^{\prime}$ and $h \circ \mu(f)=\int f d(h \circ \nu)$, for every $f \in C(X)$.

Now we will prove that $C(X) \subset L^{1}(\nu)$. Let $S$ be the closed unit ball of $C_{b}(X)$. Fix an $f \in C(X), f \geq 0$, and $A \in \mathcal{B}_{0}$. Take a net $\left\{g_{\alpha}\right\} \subset S$ such that $\int\left|g_{\alpha}-\chi_{A}\right| d|\lambda| \rightarrow 0$, for every $\lambda \in M_{\sigma}(X)$. Now $\left\{g_{\alpha} f\right\}$ is order-bounded in $C(X)$ and so $\left\{\mu\left(g_{\alpha} f\right)\right\}$ in relatively weakly compact in $E$. By taking subsets, if necessary, assume $\mu\left(g_{\alpha} f\right) \rightarrow x \in E$ weakly. Fix an $h \in E^{\prime}$. We have $h \circ \mu\left(g_{\alpha} f\right) \rightarrow h(x)$ and so $\int\left(g_{\alpha} f\right) d(h \circ \nu) \rightarrow h(x)$. Now since $f$ is integrable 
with respect to $|h \circ \nu|$, we get $\int\left(g_{\alpha} f\right) d(h \circ \nu) \rightarrow \int_{A} f d(h \circ \nu)$. From this we get that $\int_{A} f d(h \circ \nu)=h(x), \forall h \in E^{\prime}$. This implies that $f \in L^{1}(\nu)$. Now, from $\int f d(h \circ \nu)=h \circ \mu(f), \forall h \in E^{\prime}$, it follows that $\int f d \nu=\mu(f)$. We denote $\nu$ by $\mu$.

(ii). Fix a $p \in P$. By the mapping $x \rightarrow\{f(x)\}_{f \in C(X)}, X$ can be imbedded in $R^{C(X)}$, with product topology. Denoting $[-\infty, \infty]$ by $\bar{R}$, we get that $X$ is embedded in the compact Hausdorff space $\bar{R}^{C(X)}$ (with product topology). The closure of $X$, in $R^{C(X)}$, is the real-compactification of $X$ and will be denoted by $v X$; the closure of $X$, in $\bar{R}^{C(X)}$, is the Stone-Cech compactification and will be denote by $\tilde{X}$. Every $f \in C(X)$ extends continuously to $v X$ (it will be real-valued; just the component-wise values); it also extends continuously to $\tilde{X}$ (can have values $\pm \infty$; just the component-wise values).

We will complete the proof (ii) in several steps:

I. For an $f \in C(X)$, there is a $c \geq 0$ such that if $U=\{x \in X:|f(x)|>c\}$ then $\bar{\mu}_{p}(U)=0$.

Proof. The result will be proved if we prove under the assumption that $f \geq 0$. Suppose $\bar{\mu}_{p}\left(W_{n}\right)>0, \forall n \in N$, where $W_{n}=\{x \in X: \mid f(x)>n\}$. Then there are sequences $\left\{a_{n}\right\}$ and $\left\{b_{n}\right\}$ of positive real numbers such that, for every $n$, $a_{n}<b_{n}<a_{n+1}, \lim a_{n} \rightarrow \infty$ and $\bar{\mu}_{p}\left(U_{n}\right)=c_{n}>0$, where $U_{n}=f^{-1}\left(a_{n}, b_{n}\right)$. Take a sequence $\left\{h_{n}\right\} \subset E^{\prime}$ such that $\left|h_{n}\left(V_{p}\right)\right| \leq 1$ and $\left|h_{n} \circ \mu\right|\left(U_{n}\right)>c_{n}, \forall n$. Choose $\left\{g_{n}\right\} \subset C_{b}(X), 0 \leq g_{n} \leq \chi_{U_{n}}$ such that $\left|h_{n} \circ \mu\left(g_{n}\right)\right|>c_{n}, \forall n$. Let $f_{0}=\sum \frac{n}{c_{n}} g_{n} f$. Then $f_{0} \in C(X)$ and $\left|h_{n} \circ \mu\right|\left(f_{0}\right) \geq \frac{n}{c_{n}}\left|h_{n} \circ \mu\left(g_{n}\right)\right| \geq n, \forall n$. Since $f_{0} \in L^{1}(\nu)$, this is a contradiction. The smallest such $c$ (which will exist because of countable additivity) will be denoted by $c_{f}$.

II. Let $\tilde{\mu}$ be the regular Borel measure on $\tilde{X}$ associated with $\mu \in M_{\sigma}(X, E)$. For an $f \in C(X)$, let $A_{f}=\left\{x \in X:|f(x)| \leq c_{f}\right\}$ ( $c_{f}$ is defined in I). Then $\overline{\tilde{\mu}}_{p}\left(\tilde{X} \backslash \overline{A_{f}}\right)=0$.

Proof. Suppose this is not true. Then there is an $h \in E^{\prime}$ with $\mid h\left(V_{p} \mid \leq 1\right.$, and a $g \in C(\tilde{X})$ such that $|g| \leq \chi_{\tilde{X} \backslash \overline{A_{f}}}$ and $|(h \circ \tilde{\mu})(g)|>0$. This means $|g|_{\mid X} \leq \chi_{X \backslash A_{f}}$ and $|(h \circ \mu)(g)|>0$ which is a contradiction by $\mathrm{I}$.

III. For an $f \in C(X)$ let $\bar{f}$ be its extension to $v X$. Let $A_{\bar{f}}=\{x \in v X$ : $\left.|\bar{f}(x)| \leq c_{f}\right\}$. Then $\overline{\tilde{\mu}}_{p}\left(\tilde{X} \backslash \overline{A_{\bar{f}}}\right)=0$. Consequently, $\overline{\tilde{\mu}}_{p}\left(\tilde{X} \backslash \cap\left\{\bar{A}_{\bar{f}}: f \in C(X)\right\}\right)=$ 0 .

Proof. Since $\overline{A_{\bar{f}}} \supset \overline{A_{f}}$, the result follows by II.

IV. $\cap\left\{\overline{A_{\bar{f}}}: f \in C(X)\right\}=\cap\left\{A_{\bar{f}}: f \in C(X)\right\}$.

Proof. To prove this, take a $y \in \cap\left\{\overline{A_{\bar{f}}}: f \in C(X)\right\}$. Fix an $f \in C(X)$. Suppose $y \notin A_{\bar{f}}$; then $y \notin v X$. Take a $g \in C(X)$ such that $\bar{g}(y)=\infty$. This means $y \notin \cap \overline{A_{\bar{g}}}$. This contradiction proves the result.

V. If $C=\cap\left\{A_{\bar{f}}: f \in C(X)\right.$, then $C$ is a compact subset of $v X$ and $\overline{\tilde{\mu}}_{p}(\tilde{X} \backslash C)=0$.

Proof. It follows from IV that $C$ is compact in $v X$. Now, from III, $\overline{\tilde{\mu}}_{p}(\tilde{X} \backslash C)=$ 0 . This proves the result. 
In the following corollary we take $E$ to be an order complete locally convex vector lattice such that if a bounded net $\left\{x_{\alpha}\right\}$ order converges to $x$ then $x_{\alpha} \rightarrow x$ in $E$; these assumptions imply that $E$ is an ideal in $E^{\prime \prime}$ and order intervals in $E$ are $\sigma\left(E, E^{\prime}\right)$-compact ([1], Theorem 11.13, p. 170). By ([9], 7.5, Corollary 1), if $E$ is an order complete vector lattice whose order is regular and of minimal type, then $E$ with order topology ([9], Sec. 6, p. 230) has the above property (examples of these spaces are given in [9], p. 240).

Corollary 8. Let $E$ be an order complete locally convex vector lattice with the property if a bounded net $\left\{x_{\alpha}\right\}$ order converges to $x$ then $x_{\alpha} \rightarrow x$ in E. Let $\mu: C(X) \rightarrow E$ be a positive linear map. Then

(i) There is a unique $\nu \in M_{\sigma}(X, E)$ such that $C(X) \subset L^{1}(\nu), \nu \geq 0$ (this means $f \in C(X), f \geq 0$ implies $\nu(f) \geq 0)$ and $\mu(f)=\int f d \nu, \forall f \in C(X)$;

(ii) for every $p \in P$ there is compact $C \subset v X$ (the real-compactification of $X)$, depending on $p$, such that $\overline{\tilde{\nu}}_{p}(\tilde{X} \backslash C)=0$.

Proof. The assumptions on $\mu$ and $E$ imply that order bounded sets are mapped into relatively $\sigma\left(E, E^{\prime}\right)$-compact subsets $E$. The result follows from Theorem 7 .

Let $M_{c}(X, E)=\left\{\mu \in M(X, E): \operatorname{supp}\left(\overline{\tilde{\mu}}_{p}\right) \subset v X, \forall p \in P\right\}$. It is easy to see that $M_{c}(X, E) \subset M_{\sigma}(X, E)$ : Take a $\mu \in M_{c}(X, E)$ and a bounded sequence $\left\{f_{n}\right\} \subset C_{b}(X), f_{n} \rightarrow 0$, pointwise to 0 in $X$; this means $f_{n} \rightarrow 0$, pointwise on $v X$ (well-known result). Now $\mu\left(f_{n}\right)=\tilde{\mu}\left(\tilde{f}_{n}\right) \rightarrow 0$ implies that $\mu \in M_{\sigma}(X, E)$.

The following corollary is somewhat converse to Theorem 7 ; it says that measures in $M_{c}(X, E)$ map order-bounded subsets of $C(X)$ into relatively weakly compact subsets of $E$.

Corollary 9. Let $\mu \in M_{c}(X, E)$. Then $C(X) \subset L^{1}(\mu)$ and in the linear map $\mu: C(X) \rightarrow E$, order-bounded subsets are mapped into relatively weakly compact subsets of $E$.

Proof. Take an $f \in C(X), f \geq 0$. Fix a $p \in P$ and let $C=\operatorname{supp}\left(\overline{\tilde{\mu}}_{p}\right.$. Put $M=\sup \tilde{f}(C) . U=\{x \in \tilde{X}: \tilde{f}(x)>M\}$ is an open Baire set in $\tilde{X}$ and is disjoint from $C$ so that $\overline{\tilde{\mu}}_{p}(U)=0$. From this it easily follows that $\bar{\mu}_{p}(U \cap X)=0$ (note $U \cap X$ is a Baire set in $X$ ). Also, $f \leq M$ a.e. $\left[\lambda_{p}\right]$. Since the constant functions are in $L^{1}(\mu)$, by ([7], Theorem 2, p. 30), $f \in L^{1}(\mu)$.

Putting $h=\inf (f, M)$, we have $f=h$ a.e. $\left[\lambda_{p}\right]$. Let $K$ be an absolutely convex, weakly compact subset of $E$ such that $\mu(S) \subset K(S$ being the closed unit ball of $\left.C_{b}(X)\right)$. This means $\mu(h) \in M K$. Since $f=h$ a.e. [ $\left.\lambda_{p}\right]$, we have $\mu(f) \in M K$. This proves that order-bounded sets are mapped into relatively weakly compact sets.

\section{ACKNOWLEDGEMENT}

We are very thankful to the referee for pointing out some typographical errors and also for making some very useful suggestions which have improved the paper. 


\section{REFERENCES}

1. C. D. Aliprantis and O. Burkinshaw, Positive operators. Pure and Applied Mathematics, 119. Academic Press, Inc., Orlando, FL, 1985.

2. J. Diestel and J. J. Uhl, JR., Vector measures. Mathematical Surveys, No. 15. American Mathematical Society, Providence, R.I., 1977.

3. L. Drewnowski, Topological rings of sets, continuous set functions, integration. I, II. Bull. Acad. Polon. Sci. Sér. Sci. Math. Astronom. Phys. 20(1972), 269-276; ibid. 20(1972), 277-286.

4. E. Hewitt, Linear functionals on spaces of continuous functions. Fund. Math. 37(1950), 161-189.

5. S. S. Khurana, Topologies on spaces of vector-valued continuous functions. Trans. Amer. Math. Soc. 241(1978), 195-211.

6. S. S. Khurana, Topologies on spaces of vector-valued continuous functions. II. Math. Ann. 234(1978), No. 2, 159-166.

7. I. KluváaneK and G. Knowles, Vector measures and control systems. North-Holland Mathematics Studies, Vol. 20. Notas de Matemática, No. 58. [Notes on Mathematics, No. 58]. North-Holland Publishing Co., Amsterdam-Oxford; American Elsevier Publishing Co., Inc., New York, 1976.

8. D. R. LEwIS, Integration with respect to vector measures. Pacific J. Math. 33(1970), 157-165.

9. H. H. Schaeffer, Topological vector spaces. Springer Verlag, 1986.

10. F. D. Sentilles, Bounded continuous functions on a completely regular space. Trans. Amer. Math. Soc. 168(1972), 311-336.

11. R. F. Wheeler, A survey of Baire measures and strict topologies. Exposition. Math. $\mathbf{1}(1983)$, No. 2, 97-190.

12. Varadarajan, V. S., Measures on topological spaces. (Russian) Mat. Sb. (N.S.) 55 (97)(1961), 35-100; English transl.: Amer. Math. Soc., Transl., II. Ser. 48(1965), 161228.

(Received 2.11.2005; revised 21.06.2006)

Author's address:

Department of Mathematics

University of Iowa

Iowa City, Iowa 52242

U.S.A.

E-mail: khurana@math.uiowa.edu 